\title{
Characteristics of Nano-reactor and Phenomena during Mechanical Milling of Hematite-Graphite Mixture
}

\author{
Yoshiaki KASHIWAYA, Hiroshi SUZUKI') and Kuniyoshi ISHII \\ Graduate School of Engineering, Hokkaido University, Sapporo 060-8628 Japan. E-mail: Yoshiaki@eng.hokudai.ac.jp \\ 1) Student of Graduate School of Engineering, Hokkaido University, Sapporo 060-8628 Japan.
}

(Received on May 24, 2004; accepted in final form on July 26, 2004)

\begin{abstract}
The mechanical milling of hematite and graphite mixture could accelerate the reaction rate extremely, when the mixture after milling was heated up under inert atmosphere. In the previous study, the authors have indicated not only the high reaction rate but also the low starting temperature of the reaction. These phenomena can be understood through the view point of the nano-reactor and the active complex. The nano-reactor was defined in this study through the observation of the milling products by TEM and FE-SEM. The important characteristic of nano-reactor was proposed.

Moreover, the phenomena such as the reduction reaction, the phase transformation(formation of an active complex) and the crystalline size degradation occurring in the course of the milling were examined. It was found that the milling of mixture gave a different phenomenon from the single hematite or graphite milling. Furthermore, the atmosphere during the milling affected on the reactivity of the milling product. The existence of oxygen caused the reoxidation of the reduction product which resulted in the different crystalline size after milling.
\end{abstract}

KEY WORDS: nano-reactor; hematite; graphite; composite pellet; mechanical milling; ball milling; reduction; carbothermic reduction.

\section{Introduction}

Many applications of the mechanical milling (mechanical alloying, ball milling or grinding) on the chemical reaction system (so called mechanochemistry), which related to carbonaceous material, have been reported. ${ }^{1-21)}$ At the same time, the mechanochemistry of hematite (iron oxides) were investigated extensively. ${ }^{22-30)}$ On the other hand, the carbothermic reaction between hematite (iron oxides) and carbon was the subject of the iron making field for a long time. $^{31-36)}$

In previous study, ${ }^{37)}$ the authors have investigated the reactivity of hematite-graphite mixture obtained by the mechanical milling. The rate of reaction has remarkably increased and the starting temperature of reaction decreased in accordance with the milling time.

A number of the affecting factors on the reactivity of the mixture will exist. Furthermore, as the factors might interact each other, the apparent result would be complicate extremely. For example, since the grindability of the sample will strongly affect the properties of sample powder after milling, the property of crystal such as the size, the distribution and the hardness, which related to the nature of a single crystal itself and a polycrystalline particle, would result in the variety of the reactivity after milling.

To understand the reaction behavior obtained from mechanical milling, it would be very important to clarify the phenomena occurring in the course of milling. It could easily imagine the importance of the impact energy and the fre- quency, so that the rotation speed, the weight of ball and the number of ball were all important factors to be control the milling effect. In addition to these factors, the quality of material and the surface structure (roughness) of ball and vessel for milling were also unignorable factors. In the series of present study, the effect of these factors are investigating systematically and the results will be reported in near future.

In this study, the phenomena occurring in the course of milling such as the gas evolution, reduction reaction and phase transformation (formation of active complex) were investigated.

\section{Experimental}

Figure 1 shows the preparing procedure of hematite and graphite samples. Reagent hematite $\left(>99.0 \% \mathrm{Fe}_{2} \mathrm{O}_{3}\right)$ was compressed into the tablet with $25 \mathrm{~mm} \phi \times 5 \mathrm{~mm}$ thickness. The tablet was sintered at $1000^{\circ} \mathrm{C}$ for $24 \mathrm{~h}$ in air atmosphere. Then the sintered tablet was crushed roughly and adjusted the particle size distribution from 500 to $75 \mu \mathrm{m}$ using sieve before mechanical milling start. The graphite was high density $\left(1.77 \mathrm{~g} / \mathrm{cm}^{3}\right)$ and high purity $(<100 \mathrm{ppm}$ ash content). The particle size was also adjusted with the same range as the hematite. The detail of milling condition was the same as the previous study. ${ }^{37)}$ The ratio of carbon to hematite was constant $(\mathrm{C} / \mathrm{O}=1.1)$, which was the $19.84 w t \% \mathrm{C}-80.16 \mathrm{wt} \% \mathrm{Fe}_{2} \mathrm{O}_{3}$. This ratio means the $5 \%$ excess carbon for the reduction by solid carbon, when the following reaction proceeds completely. 


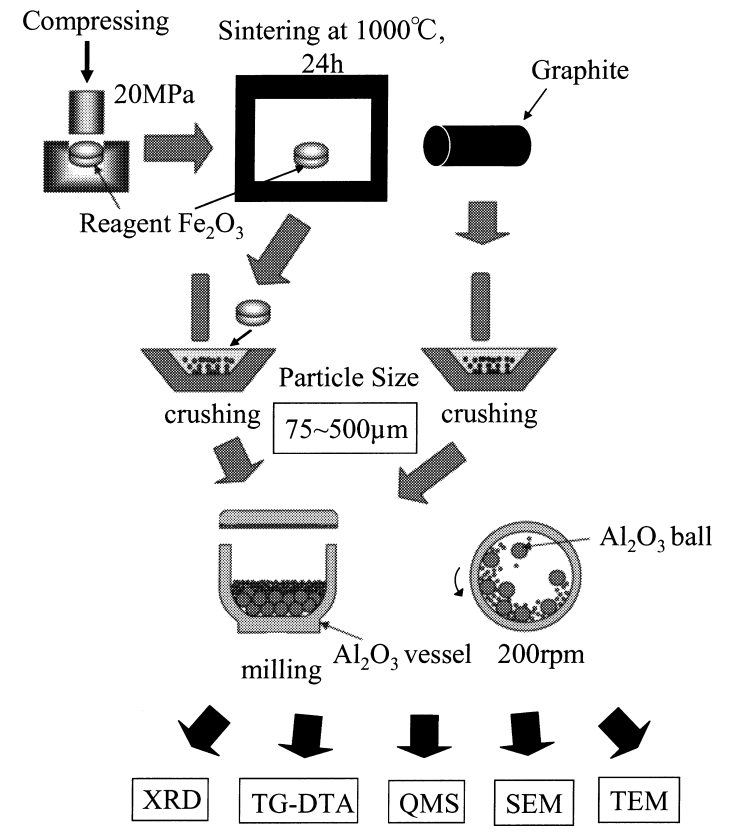

Fig. 1. Procedure of the sample preparation.

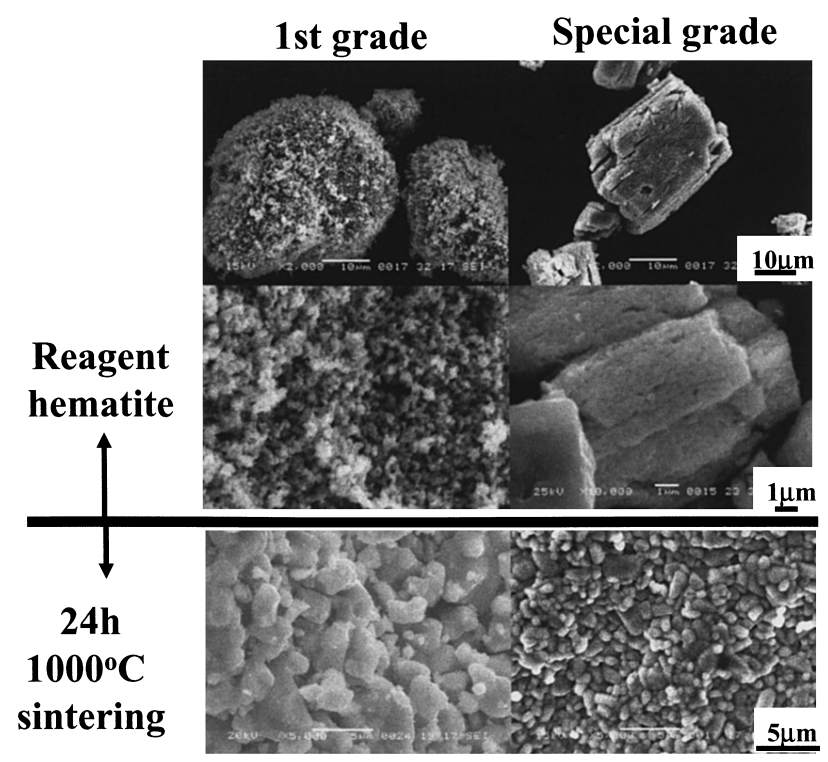

Fig. 2. SEM observation of reagent and sintered hematite particles.

$$
\mathrm{Fe}_{2} \mathrm{O}_{3}+3 \mathrm{C}=2 \mathrm{Fe}+3 \mathrm{CO}
$$

The mixture was milled in the alumina vessel $(500 \mathrm{cc})$ with ten alumina balls (weight was $17-24 \mathrm{~g}$ and diameter was $20-23 \mathrm{~mm} \phi$, which had relatively large tolerance and gradually decreased with milling time). The quality of alumina was a dense $\left(3.9 \mathrm{~g} / \mathrm{cm}^{3}\right)$ and high purity $\mathrm{Al}_{2} \mathrm{O}_{3}$ $(>99.6 \%)$.

Figure 2 shows the SEM image of the different kind of reagent hematite samples. The Special grade hematite was used in the present experiment. The special grade reagent hematite shows the polycrystalline particle (from 1 to $20 \mu \mathrm{m}$ ) consisting of smaller crystalline from 0.1 to $0.2 \mu \mathrm{m}$. On the other hand, 1st Grade hematite seems to be quite fine particle around $0.2 \mu \mathrm{m}$ and agglomerates into a larger unit around 10 to $100 \mu \mathrm{m}$. The sintering effect on the crystal morphology was also different from the property of

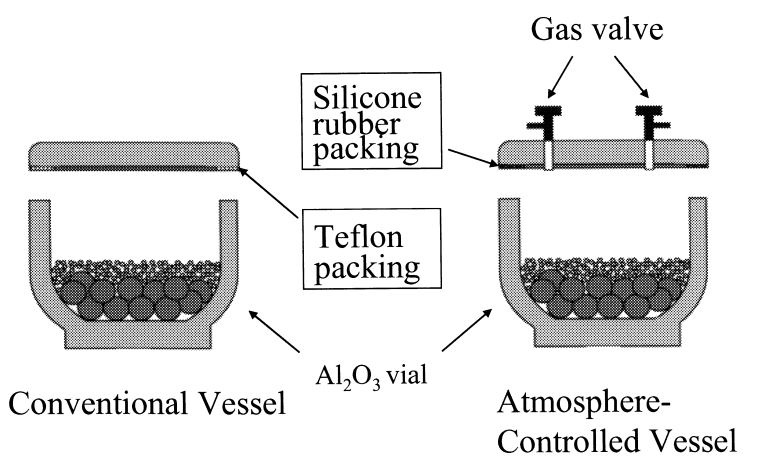

Fig. 3. Comparison between the conventional vessel and the gas tight vessel for mechanical milling.

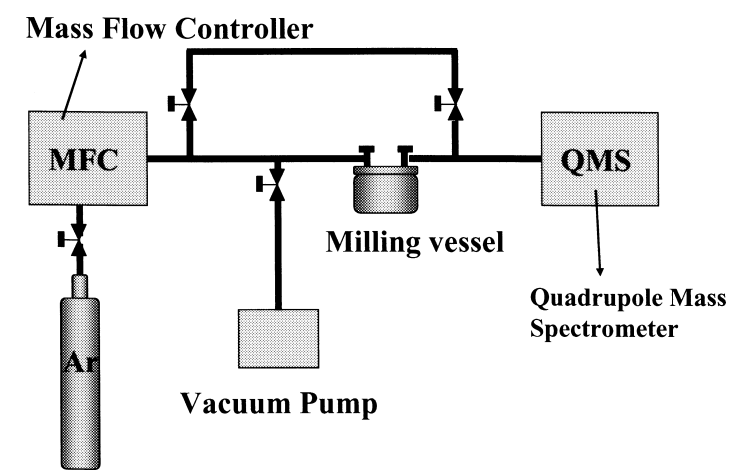

Fig. 4. Schematics of gas analysis system for the residual gas in the vessel.

starting hematite nature. The lower pictures in Fig. 2 show the morphology of hematite after $24 \mathrm{~h}$ sintering at $1000^{\circ} \mathrm{C}$. It was found that the special grade hematite showed the tendency of the hard growing crystal. As mentioned above, the properties of iron oxide affected the grindability of a powder, that resulted in the different kind of reactivity of powder.

Figure 3 shows the schematics of alumina vessels. As the teflon packing is a lower gas tight one, the silicone rubber packing was developed for atmosphere controlled vessel in this experiment. Figure 4 shows the gas analyzing system for the residual gas in the vessel after the milling. Before experiment, the atmosphere controlled vessel was set in the system, and evacuated the gas remained both in the vessel and in the tubing system. Then argon was flowed through the vessel and the residual oxygen in the argon gas was monitored by QMS (Quadrupole Mass Spectrometer). When the residual oxygen in argon decreased to the original level $(<100 \mathrm{ppm})$, the valves on the cap were closed and the vessel was moved to the milling device. After the milling, the vessel was set on this gas analyzing system again. Before opening the valves, the gas tubing system was evacuated completely by rotary pump, and then the argon gas was substituted. This procedure was repeated twice, because it was quite difficult to eliminate the residual oxygen from the long tubing system.

\section{Results and Discussions}

\subsection{Definition of Nano-reactor}

The difference between the Micro-reactor and the Nano- 


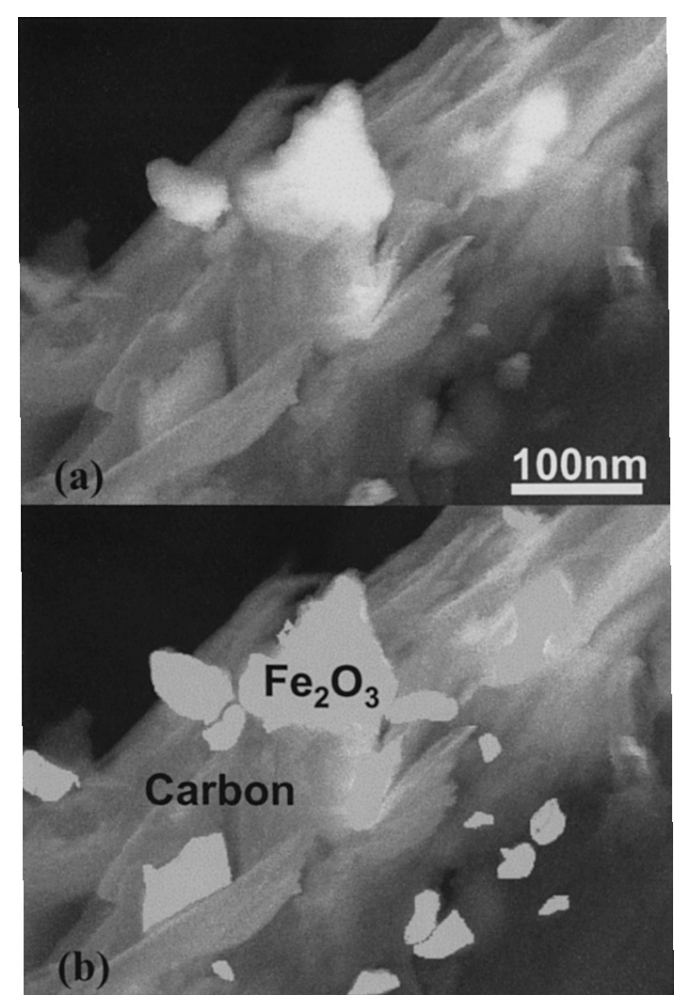

Fig. 5. Surface structure of hematite-carbon composite by FESEM image. (6h milling)

reactor is just classified by its size. Micro-reactor was defined from 100 to $1 \mu \mathrm{m}$ and Nano-reactor was less than $1 \mu \mathrm{m}$ in present study.

The Nano-reactor is not merely the mixture of hematite and carbon. The most important feature is the contacting mode between the hematite and the carbon.

Figure 5 shows the surface structure of hematite nanoparticles on the graphite particle (observation by FE-SEM), which were introduced by $6 \mathrm{~h}$ mechanical milling with $200 \mathrm{rpm}$ that was relatively low crushing energy. These nano-particles were not a perfect Nano-reactor, because the contact area was small and weak, so that the interface will separate soon, when the reaction proceeds. Furthermore, this imperfection was confirmed from the aspect of reactivity that measured using TG-DAT under argon and heating up condition, in which the $6 \mathrm{~h}$ milling sample did not show the high reactivity. ${ }^{37)}$

Figure 6 shows the internal structure of hematite-carbon composite consisted of many nano-reactors observed by TEM. The sample was milled for $72 \mathrm{~h}$. It was difficult to confirm the hematite particle from surface (by SEM), which meant the most of hematite particles were enwrapped with graphite or the particles stuck into the graphite particle. From diffraction pattern shown in Fig. 6(b), all spots were listed in the Table 1 and compared with the data from literature (ASTM card). It was found that iron oxides (hematite $\left(\mathrm{Fe}_{2} \mathrm{O}_{3}\right)$, maghemite $\left(\gamma-\mathrm{Fe}_{2} \mathrm{O}_{3}\right)$, magnetite $\left(\mathrm{Fe}_{3} \mathrm{O}_{4}\right)$ and wustite $\left.(\mathrm{FeO})\right)$ and graphite coexisted and the nano-particles of hematite were surrounded by the graphite. The gray area in the dark field image corresponds to the graphite (002) and the bright small spots means the hematite (104). As shown in previous paper, ${ }^{37)}$ the reactivity of $72 \mathrm{~h}$ milling sample shown in Fig. 6(a) was an extremely high. On the other hand, the sample shown in Fig. 5

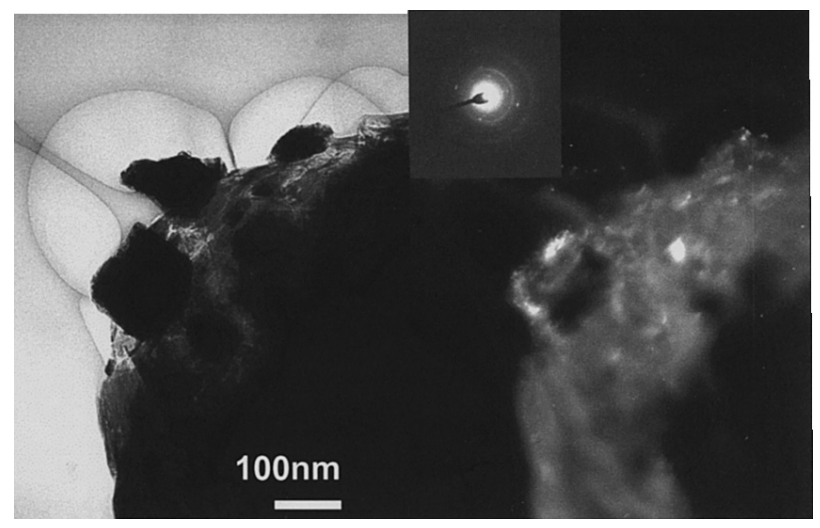

(a) Bright field image

(b) Dark field image (Hematite (104) and ring pattern of (72 hours milling) graphite (002) included)

Fig. 6. Internal structure of hematite-carbon composite consisting of nano-reactor observed by TEM.

Table 1. Identification of compounds found in the $72 \mathrm{~h}$ milling sample, which were observed by TEM.

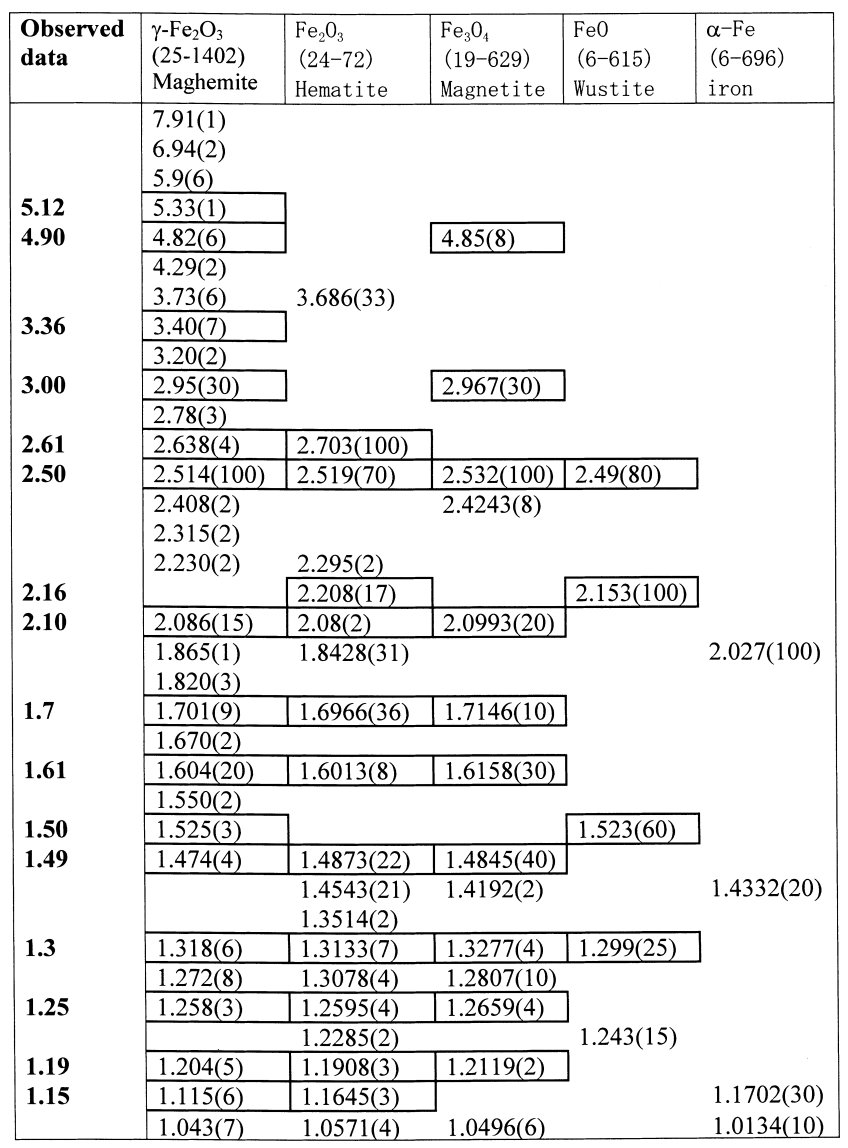

did not reach $100 \%$ reduction degree and the maximum rate of reduction was smaller.

From these results, the preconditions of the nano-reactor were as follows;

(1) The size of hematite was less than $1 \mu \mathrm{m}$.

(2) The hematite particle should be surrounded by carbon or struck into the carbon particle.

These preconditions are very important and can not attain only by a mixing of nano-particles. It would be very important that both the particles should meet with large pressure and the harder one (hematite) struck into softer one 


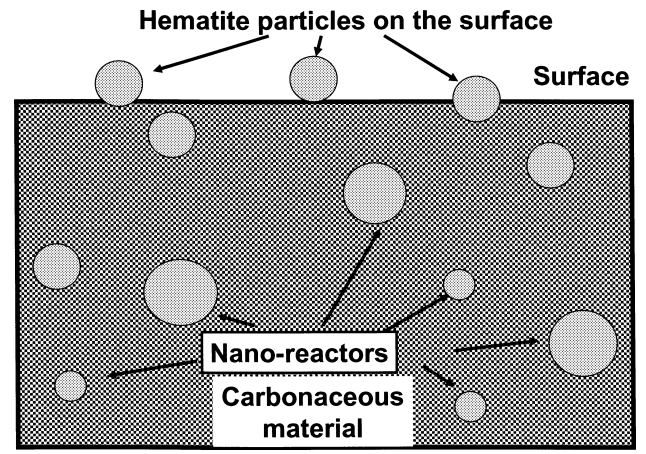

Fig. 7. Model of nano-reactor consisted of hematite and carbon, which was made by the mechanical milling.

(graphite).

Figure 7 shows the model of nano-reactor for the high rate reduction of hematite-graphite mixture. As mentioned above, the hematite particles on the graphite surface is not a perfect nano-reactor, because the contact between the hematite and graphite is easily separated during reaction. On the other hand, the hematite nano-particles in the graphite particle (or surrounded by the graphite) are able to maintain the contact until the reaction complete. This is quit important feature of the nano-reactor.

\subsection{Phenomena Occurring in the Course of Milling}

Figure 8 shows the comparison of XRD (X-ray diffraction) patterns of the samples after each millings. Fig. 8(a) shows the XRD results of the hematite-graphite mixture until $78 \mathrm{~h}$ milling and Fig. 8(b) corresponds to the results of the only hematite milling until $24 \mathrm{~h}$. From Fig. 8(a), the peak corresponding to the graphite (002) (described as $(002)_{\mathrm{G}}$ ) shows the decrease of the crystalline size (the increasing of the half-maximum width of peak and the decreasing of height of peak) with the increasing milling time. On the other hand, the hematite crystalline size showed the different degradation characteristics between the milling of mixture and the single hematite milling. In the case of the milling of single hematite (Fig. 8(b)), every peaks of XRD seemed to be lower and wider together with the milling time, while the hematite in the mixture showed the higher peaks according to the milling time, which meant the crystalline size increased or did not change during the milling. Moreover, the way of decrease on the peaks seemed to be different from the different plane. The detail of the crystalline size degradation was calculated by the Scherrer's equation $^{38)}$ and discussed in later section.

Figure 9 shows the variations of crystalline size which were calculated from graphite (002) and (101). The peak from (002) means the thickness of stacking layer (Lc) of hexagonal net plane and the one from (101) means the width of the net plane (La). ${ }^{38)}$ Actually, every crystalline decreased with milling time, almost constantly. When only graphite $(\boldsymbol{\Delta})$ was milled under an air atmosphere, both of the crystalline sizes from (002) and (101) decreased monotonously with milling time. The size of Lc decreased rapidly in comparison with the La. While in the milling of mixture, the slope of decreasing curve was relatively low under the both of the argon ( $\mathbf{\square})$ and the air atmosphere (O), although the initial decreasing rate until $20 \mathrm{~h}$ was relatively high. In

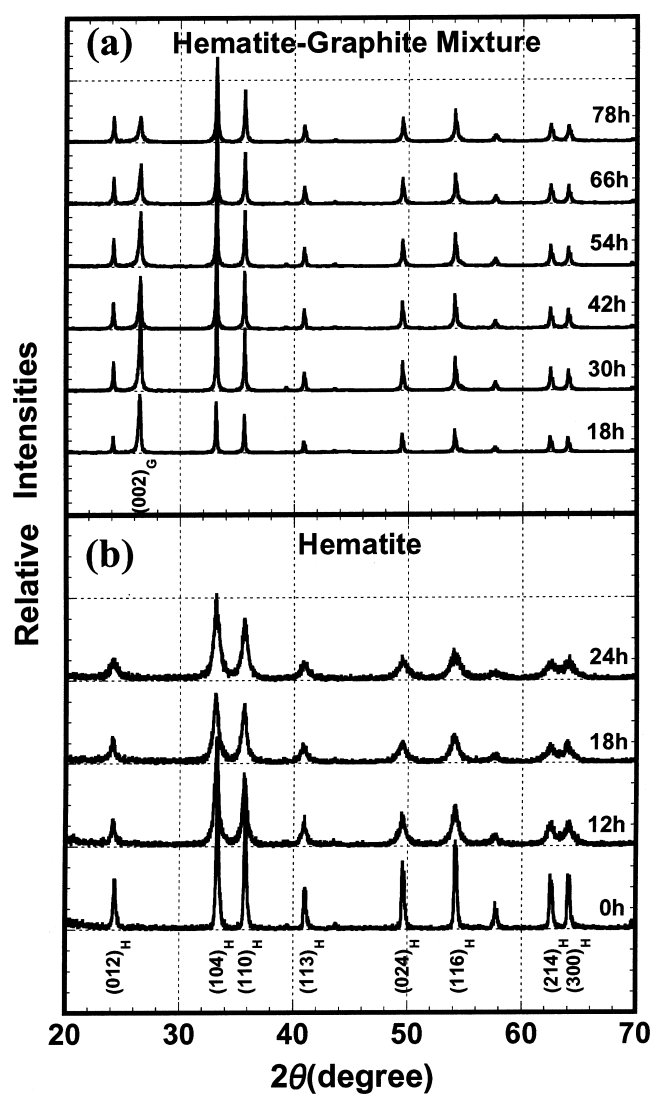

Fig. 8. Comparison of XRD patterns of milled sample in different milling time.

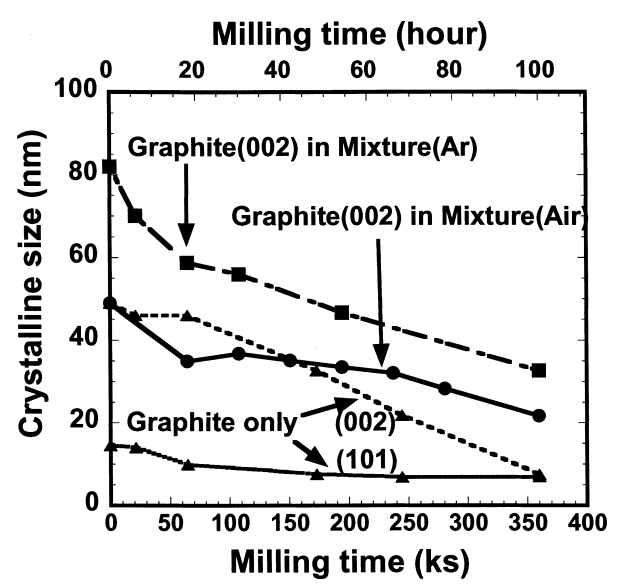

Fig. 9. Variation of crystalline size of graphite milled in an air and an argon atmosphere.

Fig. 9, the kind of graphite was different between the argon and the air atmosphere, so that the size at beginning of milling was different ( $80 \mathrm{~nm}$ for argon and $50 \mathrm{~nm}$ for air). However, the decreasing rate of the crystalline size seemed to be almost the same, which meant the lower rate of decreasing than the single graphite milling.

In Fig. 10, the variations of crystalline size of hematite between the single milling and the mixture milling under an air atmosphere were compared. As shown in Fig. 8, although many peaks from hematite planes can be observed, typical peaks were selected and the crystalline sizes were calculated as shown in Fig. 10. The variation of hematite crystalline size showed quite interesting tendency. In the single hematite milling, the crystalline size in all directions 


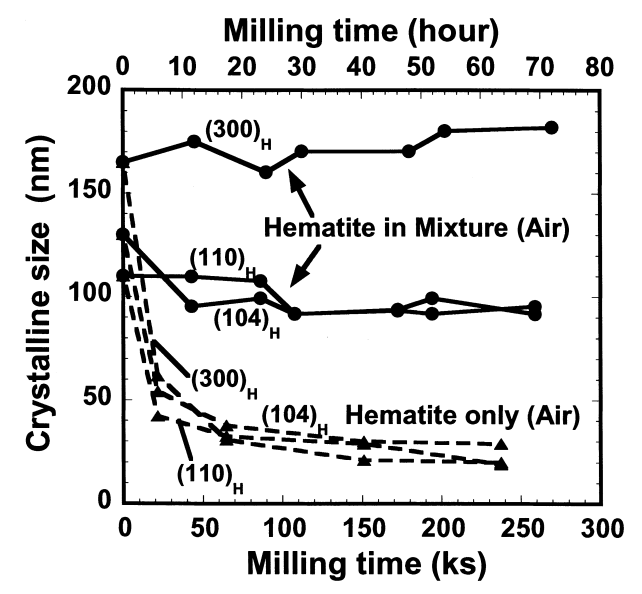

Fig. 10. Variation of crystalline size of hematite milled in an air atmosphere.

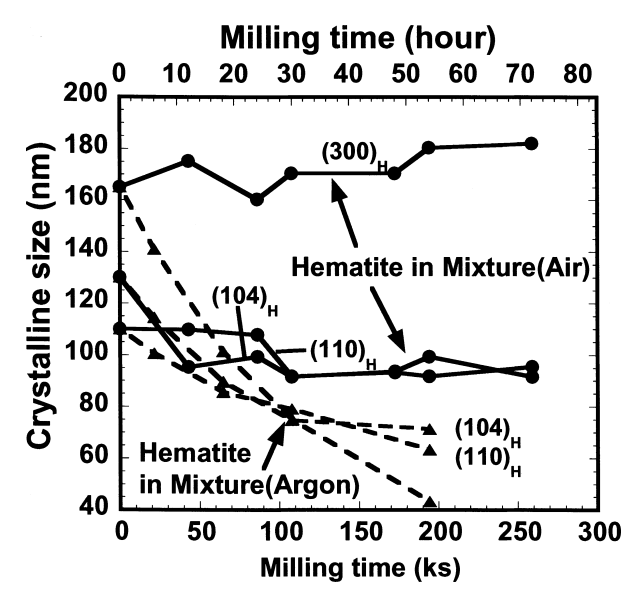

Fig. 11. Variation of crystalline size of hematite in misture milled in an air and an argon atmosphere.

(calculated from all peaks) decreased to about $30 \mathrm{~nm}$ with the milling time and the initial decreasing rate was high until $6 \mathrm{~h}$. On the other hand, in the milling of mixture, hematite crystalline size showed the different tendency from the plane. The crystalline sizes were almost the same or slightly increased in $(300)_{\mathrm{H}}$ and slightly decreased in $(110)_{\mathrm{H}}$ and $(104)_{\mathrm{H}}$, while the size from $(300)_{\mathrm{H}}$ was larger than that from $(110)_{\mathrm{H}}$ and $(104)_{\mathrm{H}}$ which was caused by the original crystal size. It was considered that the result would be caused by the reduction-reoxidation behavior of hematite during milling in an air atmosphere. In the case of reoxidation, the rate of oxidation would be different from the plane, which resulted in the different growth rate from the plane (anisotropic growth rate).

To confirm the above consideration, the milling of mixture in an argon atmosphere was carried out, in which the reoxidation will never occur. Figure 11 shows the comparison of the crystalline size variation between an air and an argon atmosphere. It was found that the crystalline size of hematite after milling in an argon atmosphere decreased continuously with the milling time.

The most of milling experiments were carried out with ten balls, and the results showed the existence of reduction during the milling from the gas analysis after milling in argon atmosphere. ${ }^{38)}$ However, in the case of the milling in air, it was difficult to confirm the reduced matter by XRD in

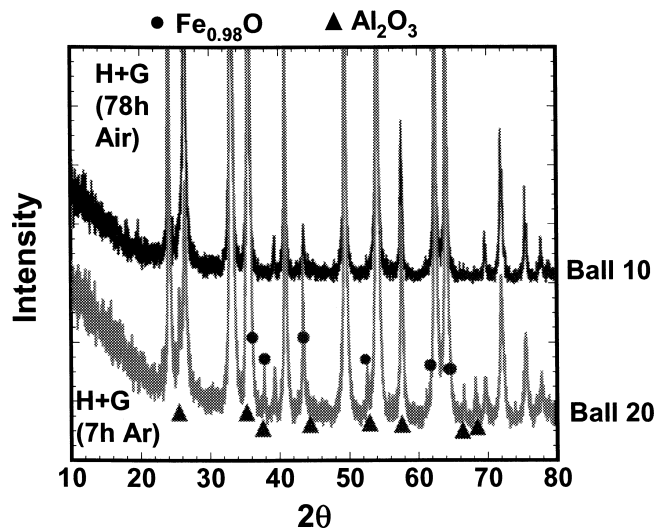

Fig. 12. Comparison of XRD patterns of the milled sample between 10 balls in air and 20 balls in argon.

order to reoxidation. Since it was expected that the amount of reduction was very small and the reactivity of the reduced one would be active, most of reduced matter was reoxidized as soon as the reduction occurred. From this reason, the milling with twenty balls was carried out for confirming the reduced substance. Figure 12 shows the results of XRD for the samples $(78 \mathrm{~h}$ milling in an air with 10 balls, $7 \mathrm{~h}$ milling in an argon with 20 balls). The reduced matter such as magnetite, wustite and iron could not find in the sample even if the milling time was $78 \mathrm{~h}$ in air. On the other hand, small quantity of wustite $\left(\mathrm{Fe}_{0.98} \mathrm{O}\right)$ was found in the sample milled in an argon, although only $7 \mathrm{~h}$ milling. Because of higher energy milling than 10 balls one, alumina was fund in the sample after milling.

As mentioned above, the reduced substance $\left(\mathrm{FeO}, \mathrm{Fe}_{3} \mathrm{O}_{4}\right)$ was found in the sample milled under the air atmosphere by TEM observation. However, it was quit small quantity to detect by XRD. Anyhow, it could be considered that the reduction would occur during milling, even in an air atmosphere with 10 balls. And these phenomena might increase the reactivity of milling product intensively ${ }^{37)}$ In addition to the simple reduction, it could be considered that the active complex which meant the medium product of reduction would exist and might affect the apparent reactively significantly. The kinetic analysis taking into account the transition state theory were performed and the active state of complex was considered in the next paper. ${ }^{39)}$

\section{Conclusions}

The milling of hematite and graphite mixture was carried out using alumina vessel under an air and an argon atmosphere. The definition of the nano-reactor was conducted for the understanding of the high reaction rate. Moreover, the phenomena occurring in the course of milling were elucidated. The obtained results are as follows:

The preconditions of Nano-reactor are

(1) The size of hematite was less than $1 \mu \mathrm{m}$.

(2) The hematite particle should be surrounded by carbon or struck into carbon particle, which support the contact between the hematite and the graphite during reaction until the reaction complete.

The phenomena occurring in the course of milling.

(3) The milling of the single hematite or graphite: The 
crystalline size decreased regardless of the atmosphere (air or argon).

(4) The milling of the mixture: The reoxidation of hematite occurred during the milling in an air atmosphere, which resulted in the anisotropic growth of hematite. On the other hand, in an argon atmosphere, the hematite crystalline size decreased constantly because of reduction.

\section{Acknowledgement}

The present study has been carried out in the research group of 'Project for the innovational ironmaking reaction in new blast furnace at half energy consumption and minimum environmental influences' supported by Special Coordination Founds of the Science and Technology Agency of the Japanese Government. The authors would like to express great appreciation to the member of research group for the valuable discussions.

\section{REFERENCES}

1) Y. Uehara: Bull. Chem. Soc. J., 48 (1975), 3383.

2) E. Kasai, K. Mae and F. Saito: ISIJ Int., 35 (1995), 1444.

3) J. Welham and J. S. Williams: Carbon, 36 (1998), 1309.

4) L.-W. Yin, M.-S. Li, Y.-X. Liu, J.-L. Sui and J.-M. Wang: J. Phys. Condens. Matter, 15 (2003), 309.

5) X. Guo, J. Qi and K. Sakurai: Scr. Mater., 48 (2003), 1185.

6) L.-W. Yin, M.-S. Li, G. Luo, J.-L. Sui and J.-M. Wang: Chem. Phys. Lett., 369 (2003), 483.

7) K. Kudaka, K. Iizumi, H. Izumi and T. Sasaki: J. Mater. Sci. Lett., 20 (2001), 1619.

8) H. L. Castricum, H. Bakker, B. van der Linden and E. K. Poels: $J$. Phys. Chem. B, 105 (2001), 7928.

9) T. Ido, M. Mori, G. Jin and S. Goto: Kagaku Kogaku Ronbunshu, 72 (2001), 121.

10) M. G. Aylmore and F. J. Lincoln: J. Alloys Compounds, 314 (2001), 103.

11) G.-L. Tan, X.-J. Wu, M.-H. Zhao and H.-F. Zhang: J. Mater. Sci., 35, No. 12, (2000), 3151

12) K. Kudaka and K. Iizumi: Mater. Technol., 17 (1999), 391.
13) B. Yao, L. Liu and W. H. Su: J. Appli. Phys., 86 (1999), 2464.

14) S. Wada, Y. Kondo, E. Sudo and Y. Maki: J. Ceram. Soc. J., 107 (1999), 611.

15) F. Miani and H. J. Fecht: Int. J. Refract. Met. Hard Mater, 17 (1999), 133.

16) G.-L. Tan and X.-J. Wu: Powder Metall., 41 (1998), 300.

17) F. N. Dzegilenko, D. Srivastava and S. Saini: Nanotechnology, 9 (1998), 330.

18) D. L. Zhang and Y. J. Zhang: J. Mater. Sci. Lett., 17 (1998), 1113.

19) N. Q. Wu, S. Lin, J. M. Wu and Z. Z. Li: Mater. Sci. Technol., 14 (1998), 287.

20) O. S. Morozova, A. N. Streletskii, I. V. Berestetskaya and A. B. Borunova: Catal. Today, 38 (1997), 107.

21) S. Mori, W.-C. Xu; T. Ishidzuki, N. Ogasawara, J. Imai and K. Kobayashi: Appl. Catal. A: General, 137 (1996), 255.

22) M. Sorescu: J. Nanoparticle Res., 2 (2000), 305.

23) W. Kim and F. Saito: Powder Technol., 114 (2001), 12.

24) G. Mi, Y. Murakami, D. Shindo, F. Saito, K. Shimme and S. Masuda: Shigen to Sozai, 115 (1999), 683.

25) J. Ding, T. Tsuzuki and G. McCormick: Nanostruct. Mater., 8 (1997), 739.

26) I. Mitov, Z. Cherkezova-Zheleva and V. Mitrov: Phys. Status Solidi (a), 161 (1997), 475.

27) T. Kosmac and T. H. Courtney: J. Mater. Res., 7 (1992), 1519.

28) J. M. Jimenez-Mateos, J. Morales and J. L. Tirado: J. Colloid Interface Sci., 122 (1988), 507.

29) E. Mendelovici, S. Nadiv and I. J. Lin: J. Mater. Sci., 19 (1984), 1556.

30) J. L. Rendon, J. Cornejo and P. De Arambarri: J. Colloid Interface Sci., 94 (1983), 546.

31) E. Kasai, T. Kitajima and T. Kawaguchi: ISIJ Int., 40 (2000), 842.

32) Q. Wang, Z. Yang, J. Tian, W. Li and J. Sun: Ironmaking Steelmaking, 24 (1997), 457.

33) C. Bryk and W.-K. Lu: Ironmaking Steelmaking, 13 (1986), 70.

34) Q. Wang, Z. Yang, J. Tian, W. Li and J. Sun: Ironmaking Steelmaking, 25 (1998), 443.

35) F. Ajersch: Can. Metall. Q., 26 (1987), 137.

36) S. K. Dutta and A. Ghosh: Metall. Trans. B, (1994), 15.

37) J. Vahdati Khaki, Y. Kashiwaya, Y. Suzuki and K. Ishii: ISIJ Int., 42 (2002), 13.

38) Y. Kashiwaya, H. Suzuki and K. Ishii: ISIJ Int., 44 (2004), 1970.

39) Y. Kashiwaya and K. Ishii: ISIJ Int., 44 (2004), 1981. 